\title{
Grief after pregnancy loss was predicted by length of pregnancy, neuroticism, psychiatric symptoms, and absence of other children
}

\author{
Janssen HJ, Cuisinier MC, de Graauw KP, et al. A prospective study of risk factors predicting grief intensity following pregnancy loss. \\ Arch Gen Psychiatry 1997 Jan;54:56-61.
}

\section{Objective}

To determine the factors that predict grief intensity in women who have a pregnancy loss.

\section{Design}

Inception cohort of women followed up for 18 months after an involuntary pregnancy loss.

\section{Setting}

The Netherlands.

\section{Patients}

2140 recently pregnant women recruited through a notice in a popular family magazine provided information on coping with normal pregnancy, delivery, and complications. 227 women $(10.6 \%)$ subsequently reported spontaneous loss of the pregnancy and 221 of these were studied. $91 \%$ of the losses occurred at $<20$ weeks of pregnancy, $97 \%$ of the women were married or in stable relationships, mean age was 29 years, $32 \%$ did not have children, and $41 \%$ had had a previous pregnancy loss. Follow up was $94 \%$.

\section{Assessment of prognostic factors}

Risk factors were assessed using the Dutch version of the Symptom Checklist-90 (psychiatric symptoms) and the Dutch Personality Questionnaire that included information on neuroticism (low self esteem, social inadequacy, general inadequacy, and aggrievedness). Information was also collected on the quality of the partner relationship, education, employment, religious background, subjectively experienced support from the woman's religion, social support, feelings about the pregnancy, conception and pregnancy variables, family demographics, and physical symptoms.

\section{Main outcome measures}

Grief and its categories (active grief, difficulty coping, and despair) measured by the Perinatal Grief Scale immediately after the pregnancy loss (mean 2.5 months) and at 6, 12, and 18 months.

\section{Main results}

All factors except previous pregnancy loss predicted grief intensity on univariate analysis. Multivariate analysis showed that grief intensity was higher for women who had been pregnant longer $(\mathrm{p}<0.001)$, had neurotic personalities before the loss $(\mathrm{p}<0.001)$, had psychiatric symptoms before the loss $(\mathrm{p}=0.02)$, and did not have other living children $(p=0.01)$. The subscales of grief intensity showed similar results for these same risk factors $(p \leqslant 0.02)$, except for the association between active grief and psychiatric symptoms before the loss $(p=0.1)$. Older women, who tended to show more grief intensity on the total Perinatal Grief Scale, showed more difficulty coping with the loss $(\mathrm{p}=0.02)$. Grief intensity, active grief, difficulty coping, and despair decreased with time ( $\mathrm{p}<0.001$ for all 4 comparisons).

\section{Conclusion}

Stronger grief responses in women who had an involuntary pregnancy loss were associated with a longer pregnancy, a more neurotic personality before the loss, psychiatric symptoms before the loss, and the absence of other living children.

Source of funding: Foundation VSB (Verenigde Spaarbank) Fund.

For article reprint:Dr HJJanssen, University of Nimegen, Department of Clinical Psychology and Persomality, Montessorilaan 3, Postbox 9104,6500 HE Nimmegen, the Netherlands. Fax +31243615594.

\section{Commentary}

Over the years several authors have examined the effects of pregnancy loss, but most of these studies have been small and frequently the sample has been drawn retrospectively from women at different stages of gestation. The study by Janssen et al represents a considerable advance on these older studies. A large number of women were recruited prospectively and follow up took place on 4 occasions over 18 months using a well validated psychometric measure. This longitudinal approach to sampling is unusual in a study of this nature.

The authors are aware of the limitations of their study. They acknowledge that participation was voluntary and this could have introduced bias and might account for the difference between their findings and those of some earlier studies. The data were gathered by self report and this could have encouraged women with psychoneurotic tendencies to respond, accounting for the apparent association between neurotic personality trait and intense grief-in fact, both might represent the same construct.

The results of the study are relevant to all nurses and other health professionals involved in women's health care in hospital and primary care settings. The findings increase our knowledge of the major factors that influence the intensity of grief experienced after pregnancy loss. In view of the robust study design it is possible to concur with the authors' conclusion that women with a more neurotic personality, a longer period of gestation before pregnancy loss, psychiatric symptomatology, and no previous living children appear to experience more grief after the loss of pregnancy than other women. Such information will be valuable to practitioners because it will enable them to identify women at particular risk of severe and prolonged grief after pregnancy loss, allowing them to tailor their interventions accordingly and to refer these women to further sources of help, such as counselling or psychiatric referral, at an early stage.

Dinah Gould, RGN, MPhil, PhD Professor of Nursing School of Health and Social Science South Bank University, London, UK 